\title{
Neural Network Model Development with Soft Computing Techniques for Membrane Filtration Process
}

\author{
Zakariah Yusuf, Norhaliza Abdul Wahab, Shafishuhaza Sahlan \\ Control \& Mechatronics Engineering, Faculty of Electrical Engineering, Universiti Teknologi Malaysia, \\ 81310 Skudai Johor Bahru, Malaysia
}

\begin{tabular}{l} 
Article Info \\
\hline Article history: \\
Received Feb 10, 2018 \\
Revised May 28, 2018 \\
Accepted May 7, 2018 \\
\hline
\end{tabular}

Keyword:

ANN modeling

GA

GSA

PSO

SMBR

\begin{abstract}
Membrane bioreactor employs an efficient filtration technology for solid and liquid separation in wastewater treatment process. Development of membrane filtration model is significant as this model can be used to predict filtration dynamic which is later utilized in control development. Most of the available models only suitable for monitoring purpose, which are too complex, required many variables and not suitable for control system design. This work focusing on the simple time seris model for membrane filtration process using neural network technique. In this paper, submerged membrane filtration model developed using recurrent neural network (RNN) train using genetic algorithm (GA), inertia weight particle swarm optimization (IWPSO) and gravitational search algorithm (GSA). These optimization algorithms are compared in term of its accuracy and convergent speed in updating the weights and biases of the RNN for optimal filtration model. The evaluation of the models is measured using three performance evaluations, which are mean square error (MSE), mean absolute deviation (MAD) and coefficient of determination (R2). From the results obtained, all methods yield satisfactory result for the model, with the best results given by IW-PSO.
\end{abstract}

Copyright $\odot 2018$ Institute of Advanced Engineering and Science. All rights reserved.

\section{Corresponding Author:}

Norhaliza Abdul Wahab,

Control \& Mechatronics Engineering Department,

Faculty of Electrical Engineering,

Universiti Teknologi Malaysia,

81310 Skudai, Johor, Malaysia.

Email:aliza@fke.utm.my

\section{INTRODUCTION}

Membrane filtration is a promising technology in many separation and purification process. This technology is able to achieve high performance in term of permeate quality. The application of membrane filtration is widely applied in many industrial applications such as water treatment and wastewater treatment process, food processing, desalination, medical application and many more. Despite the advance in the current technology, membrane filtration still experiencing numerous drawbacks in terms of fouling, high energy consumption and a significantly higher operational cost compared to conventional techniques [1].

To improve the membrane filtration process, one of the options is to obtain accurate modeling of the filtration process. With the obtained model, an accurate prediction can be made for future action of the process in order to improve the filtration performance of hydraulic cleaning involving aeration airflow, backwash, relaxation and chemical cleaning. From the obtained model, the best setting for the filtration permeate flux set point and the transmembrane pressure (TMP) dynamic behavior can also be determined. However, developing a working model for membrane filtration process is not an easy task, due to the nonlinear chaotic behavior of fouling. In literature, several mechanisms were developed to obtain an optimum filtration model. Darcy's laws for example, several terms of fouling resistance are added in series to 
represent the fouling layer in the membrane filtration process to develop the filtration model. Similar to other mechanistic or first principle model, tedious calibration process involving many parameters is required.

An alternative technique to represent a dynamic behavior of the filtration process is using artificial neural network (ANN), which has high accuracy of prediction capability. The ANN technique is employed in numerous areas including chemical process, mechanical applications, financial forecasting and trending, environmental prediction and others. On the area of membrane filtration modeling, Geissler et al [2] has developed models which are semi empirical model and ANN based model for permeate flux modeling in submerged capillary MBR. The ANN model is based on Elman neural network structure to predict the permeate flux. The techniques yield very good results, where the semi empirical model required small input variables compared to ANN. However, the ANN model gave high accuracy with the average error of $2.7 \%$.

In [3], a flat sheet submerged membrane bioreactor (SMBR) filtration for wastewater filtration is modeled using ANN model to represent the backwash effect to the permeate flux. Multilayer feed forward neural network is used to model the system. Various backwashed and filtration interval are used as the input to the model, meanwhile flux is used as the output. With the given backwashed to permeate ratio, the model is able to predict the permeate flux performance. Nevertheless, the application of ANN using conventional back propagation (BP) algorithm for training has faced several problems such as slow convergent and the algorithm has tendency to tarp in the local minima. Thus, the application of heuristic search optimization technique is one of the solutions.

Several works has found in literature to find an optimal weight and bias value in the training of the neural network. Among the widely used heuristic search algorithm for ANN model is genetic algorithm (GA).this algorithm is among the pioneer in heuristic optimization which is inspired from the population evolutionary theory. GA was successful used in [4], [5] and [6] to train the neural network model in various applications. This algorithm is able to produce a global solution for a given optimization problems. Particle swarms optimizations (PSO) in particular, have become a very effective optimization technique in various fields. The algorithm is fast and very reliable in searching for minimization. Several well known advancement of PSO algorithm such as random PSO (RPSO), constriction factor PSO (CPSO) and inertia weight PSO (IW PSO) have been developed throughout the years. These algorithms have been tested in previous neural networks application as reported in [7], [8] and [9]. Gravitational search algorithm (GSA) [10] is another type of optimization technique. This method is inspired by the law of gravity and the mass interaction in universe. The GSA was reported to yield faster convergent rate and is reliable to meet the objective function in many applications as reported in [10]-[12].

This work focuses on model development of membrane filtration process using recurrent neural network (RNN) model train by three type's of soft computing optimization techniques which are GA, PSO and GSA. These three algorithms will be used to search for the best weights and biases of the recurrent neural network model. The trained mode is then compared in term of its accuracy on the training and testing of membrane filtration data set.

\section{EXPERIMENT SETUP}

The data set is collected from the developed membrane bioreactor pilot plant located in Process Control Lab, Faculty of Electrical Engineering, Universiti Teknologi Malaysia (UTM). Random magnitude steps input were given to the suction pump to stimulate the dynamic behavior of the process. Figure 1 shows the plant schematic diagram while Figure 2 shows the data collected from the pilot plant. The experiments were carried out in single tank submerged membrane bioreactors, with working volume of $20 \mathrm{~L}$ Palm Oil Mill Effluent (POME) taken from Sedenak Palm Oil Mill Sdn. Bhd. in Johor, Malaysia. The aeration during filtration is set around 6 to 8 litter per minute (LPM) to maintain the dissolved oxygen level to be more than $2 \mathrm{ppm}$ during the experiment. In this work, voltage input of the permeate pump is used for the input, mean while the flux and TMP are the measurement outputs. 


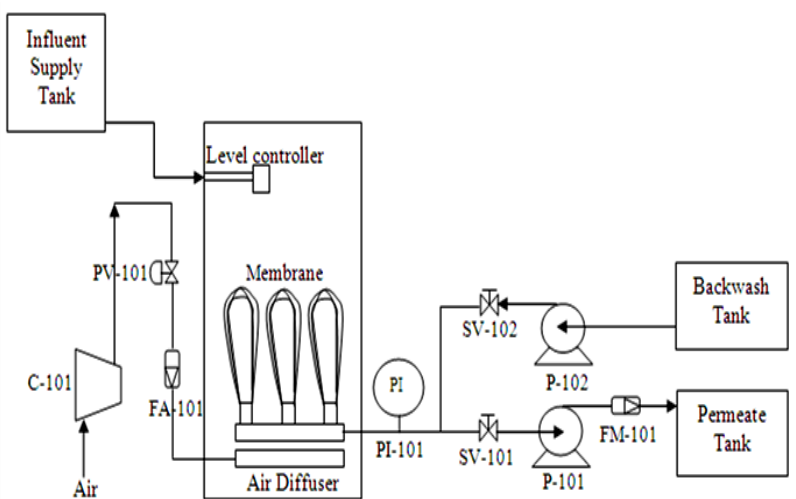

Figure 1. Plant schamatic
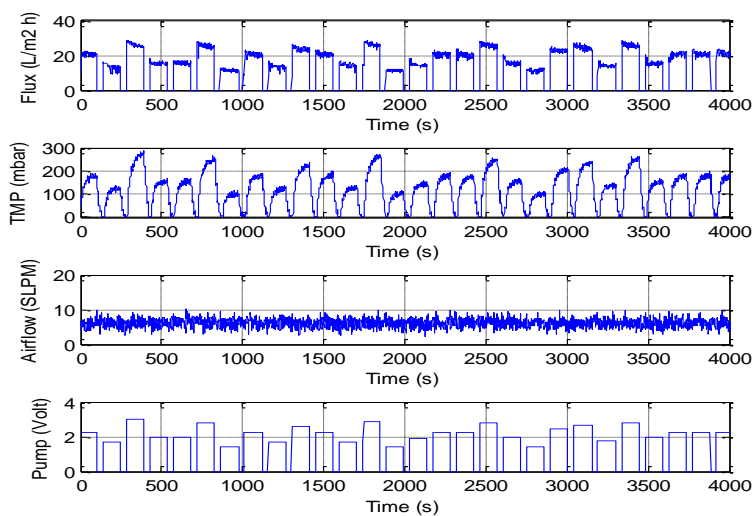

Figure 2. Experimental data

In this work, Polyethersulfone (PES) material with approximately 80-100kda pore size and a surface area of approximately $0.35 \mathrm{~m}^{2}$ is used in the filtration system

\section{MODEL DEVELOPMENT}

Recurrent neural network ( $\mathrm{RNN}$ ) model is a mathematical model developed based on the past input and past output of the system. The RNN is developed based on the basic feed forward neural network (FFNN). The training algorithm is employed to obtain suitable weights and biases of the network in order to minimize the error in the training procedure. Figure 3 shows the basic structure of the neural network model.

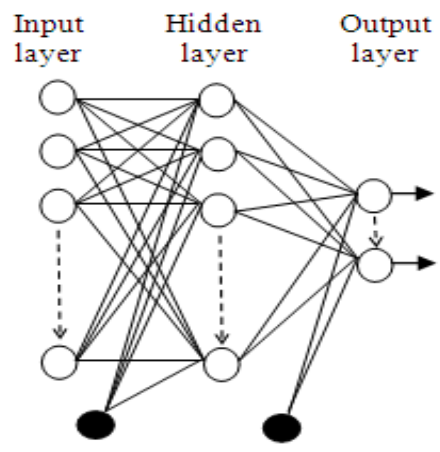

Figure 3. Neural network structure

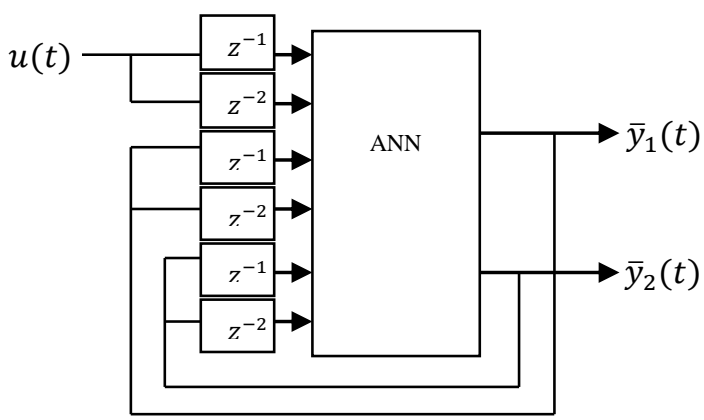

Figure 4. Recurrent neural network structure

The neural network equation can be represented as:

$$
\hat{y}_{1}(t)=E_{i}\left[\sum_{j=1}^{n_{h}} W_{i j} f_{j}\left(\sum_{l=1}^{n_{\varphi}} w_{i j}+w_{j 0}\right)+W_{i 0}\right]
$$

where $\hat{y}_{i}(t)$ is the prediction output. $F_{\mathrm{i}}$ is the function of the network, $\varphi$ is the input vector, $W_{i j}$ and $w_{i j}$ represent the network connection layer weights and biases respectively. In this work three and five hidden neurons are tested with basic three layers neural networks which are the input layer, single hidden layer and output layer. This work employed nonlinear autoregressive with exogenous input (NARX) ANN structure which shows in Figure 4. The optimization techniques applied in the ANN model training is optimize the weights and biases of the ANN structure by minimizing the mean square error (MSE) of the model

\subsection{Genetic algorithm (GA)}

Optimization algorithm is a developing area in the soft computing study. GA algorithm is one of the pioneers in evolution based optimization techniques and is a reliable technique in optimization. This 
optimization technique is widely applied in many industrial applications such as in [13] and [14]. The flow chart of the algorithm can be represented in Figure 5.

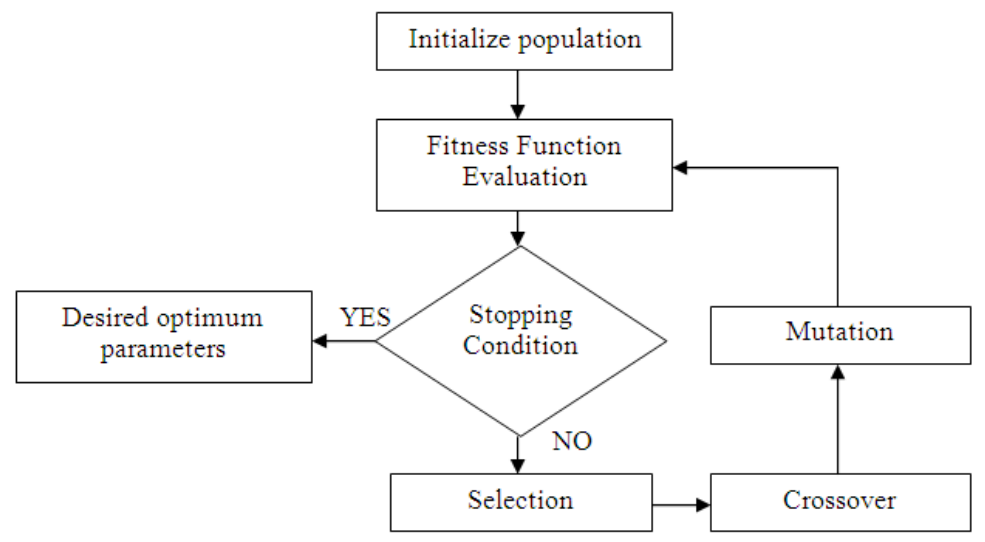

Figure 5. GA Optimization flowchart

\subsection{Particle swarm optimization}

Particle swarm optimization (PSO) is inspired by behavior of a group of animals hunting. This heuristic search optimization is very effective in finding optimal solution for many problems. In PSO algorithm, number of swarm must be selected to search for the solution. Each swarm contains an individual calls a particle. The PSO main algorithm is to update the position of each particle with an estimated velocity. Each of the components of the velocity equation represents the exploration ability and capability of individual learning as well as social learning. The PSO algorithm execution flow chart is shown in Figure 6.

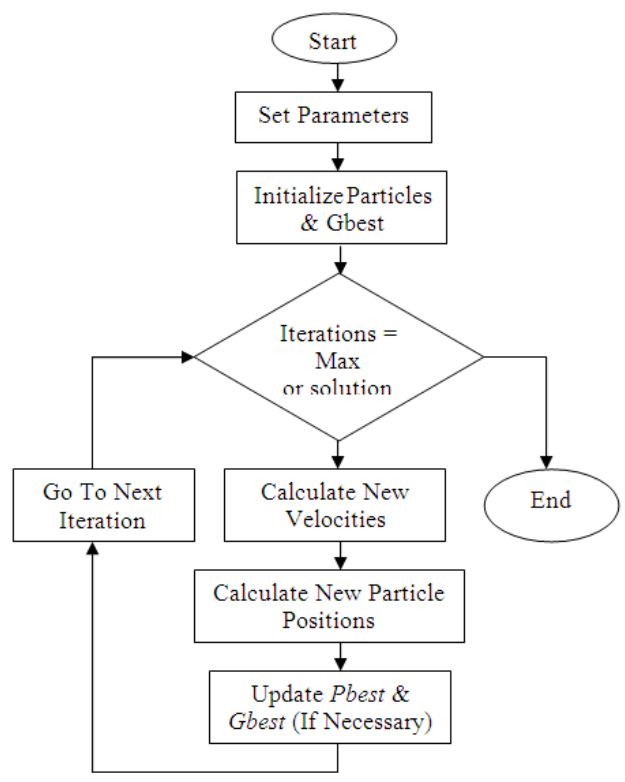

Figure 6. PSO flowchart

\subsection{Gravitational search algorithm (GSA)}

The GSA algorithm is an effective optimization algorithm developed in [10]. It is inspired from gravity law and mass interaction in the universe. Figure 7 presents the flow chart of the GSA optimization algorithm. The details development of the algorithm is presented in [10]. 


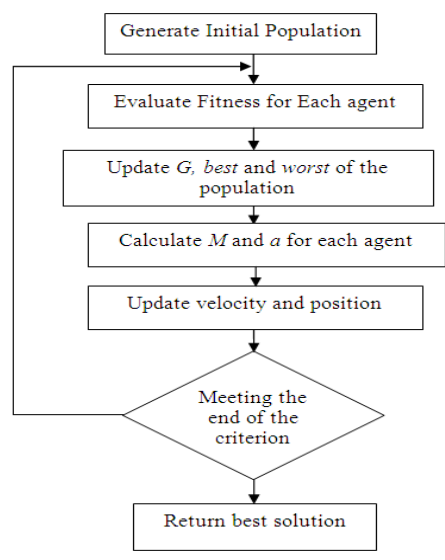

Figure 7. GSA flowchart

\section{RESULTS AND ANALYSIS}

Generally, all the training methods using soft computing techniques are able to produce acceptable result for the model obtained. From the training result it shows that PSO algorithm performs much better when compared with GA and GSA. From Figure 8(a) it can be observed that GA has a much faster searching for the right weights and biases of the neural network compared with the other. However, when approaching the first 50 iterations, PSO shows superiority in finding global minimum from the given cost function. Meanwhile, Figure 8(b) shows the zooming convergent area of the training algorithms. In term of modeling performance criteria, the PSO algorithm performs better compared with the other methods. Table 1(a) present the permeate flux training result. Table 1(b) shows the result for the TMP modeling.

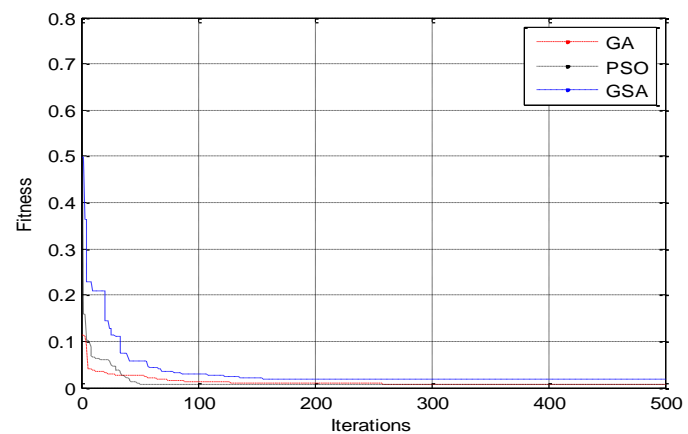

(a)

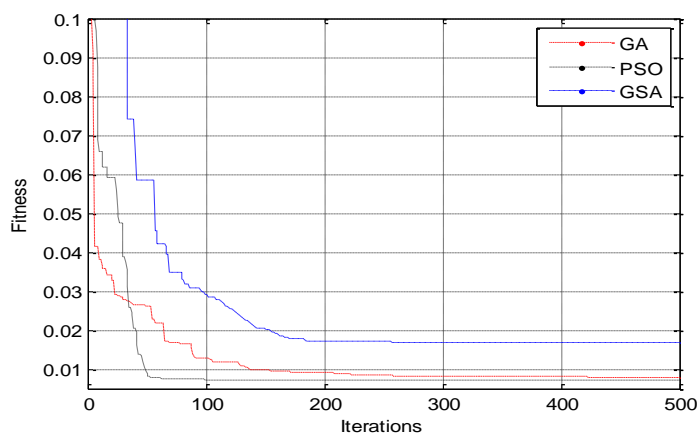

(b)

Figure 8. (a) The performance of the training algorithm (b) Zoom at the convergent area of the training algorithm

Table 1. (a) Permeate Flux Modeling Training Result

\begin{tabular}{cccc}
\hline Method & $R^{2}$ & MSE & MAD \\
\hline GA-RNN & 93.48 & 0.0066 & 0.0473 \\
PSO-RNN & 94.38 & 0.0057 & 0.0418 \\
GSA-RNN & 93.12 & 0.0070 & 0.0493 \\
\hline & & & \\
& Table 1. (b) TMP Modeling Training Result & \\
\hline Method & $R^{2}$ & MSE & MAD \\
\hline GA-RNN & 97.90 & 0.0014 & 0.0287 \\
PSO-RNN & 97.82 & 0.0015 & 0.0296 \\
GSA-RNN & 83.59 & 0.0113 & 0.0848 \\
\hline
\end{tabular}

Figure 9 (a) and Figure 9(b) shows the comparison between actual and predicted training model using PSORNN for the permeate flux and TMP. 

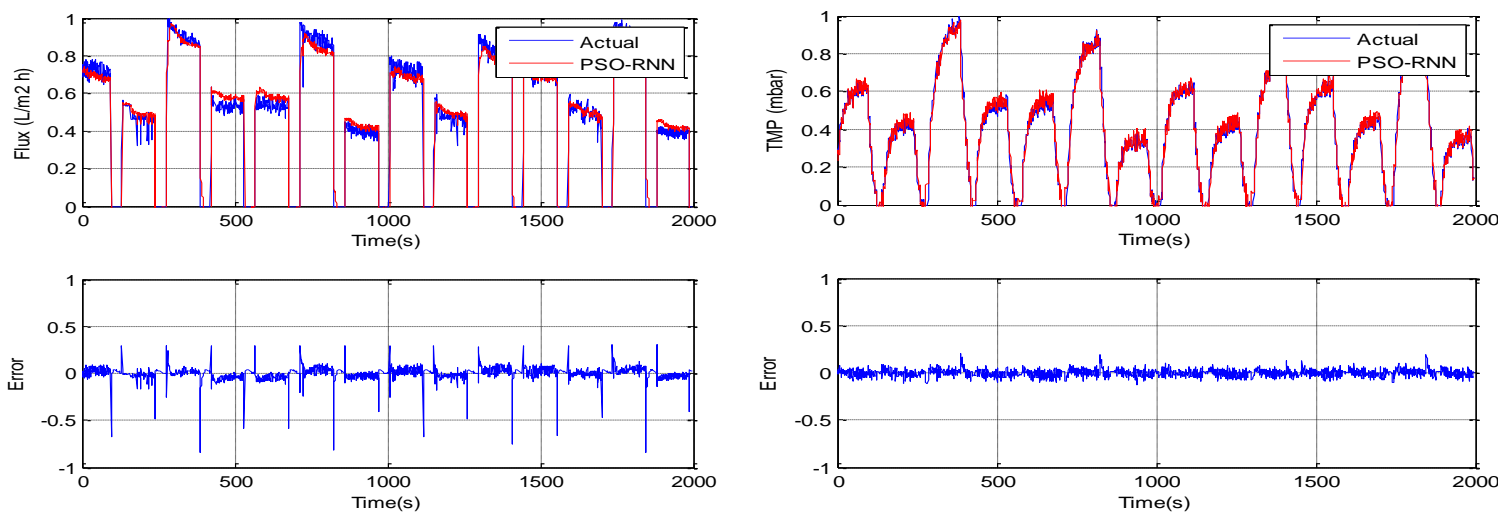

(a)

(b)

Figure 9. (a) Permeate Flux modeling training result using RNN-PSO three hidden neuron and the residuals between actual and model (b) TMP modeling training result using RNN-PSO three hidden neuron and the residuals between actual and model

The validation of the model is done by using data not utilized for the model development. From the result given in the Table 2(a) and (b), it is proven that the training performances of all the algorithms are able to model the system. Generally, the testing result is less accurate compared with the training. However, from the testing performance it showed that the models are able to replicate the dynamic of the membrane filtration system as shown in Figure 10.

Table 2. (a) Permeate Flux Modeling Testing Result

\begin{tabular}{cccc}
\hline Method & $\% R^{2}$ & MSE & MAD \\
\hline GA-RNN & 93.62 & 0.0062 & 0.0452 \\
PSO-RNN & 94.19 & 0.0056 & 0.0418 \\
GSA-RNN & 92.76 & 0.0070 & 0.0448 \\
\hline
\end{tabular}

Table 2. (b) TMP Modeling Testing Result

\begin{tabular}{cccc}
\hline Method & $\% R^{2}$ & MSE & MAD \\
\hline GA-RNN & 97.85 & 0.0013 & 0.0275 \\
PSO-RNN & 97.87 & 0.0012 & 0.0283 \\
GSA-RNN & 81.33 & 0.0115 & 0.0839 \\
\hline
\end{tabular}
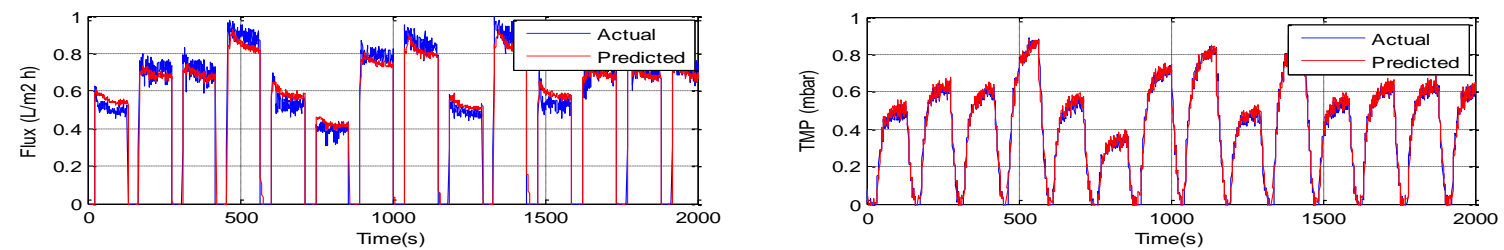

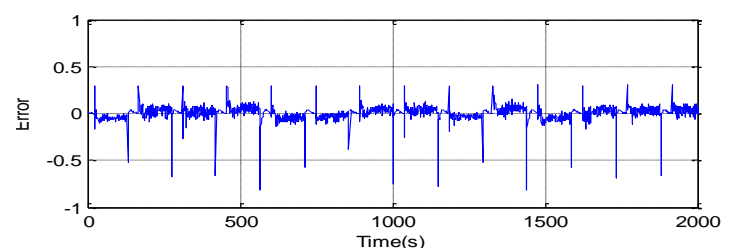

(a)

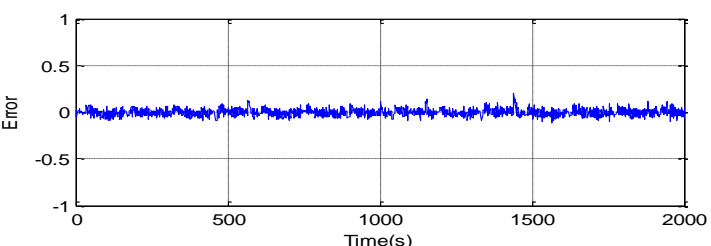

(b)

Figure 10. (a) Permeate Flux modeling testing result using RNN-PSO three hidden neuron and the residuals between actual and model (b) TMP modeling testing result using RNN-PSO three hidden neuron and the residuals between actual and model 
The result of the increment of the hidden neurons of the neural network has shown significant difference from the initial (three hidden neurons) training. It is an interesting finding where some of the training algorithm shows improvement of the model while the other is not able to find suitable weights and biases of the neural network model until the end of the iterations. Figure 11(a) shows the performance for each of the training methods in term of it fitness and iterations. Figure 11(b) shows the zoomed image at the convergent area.

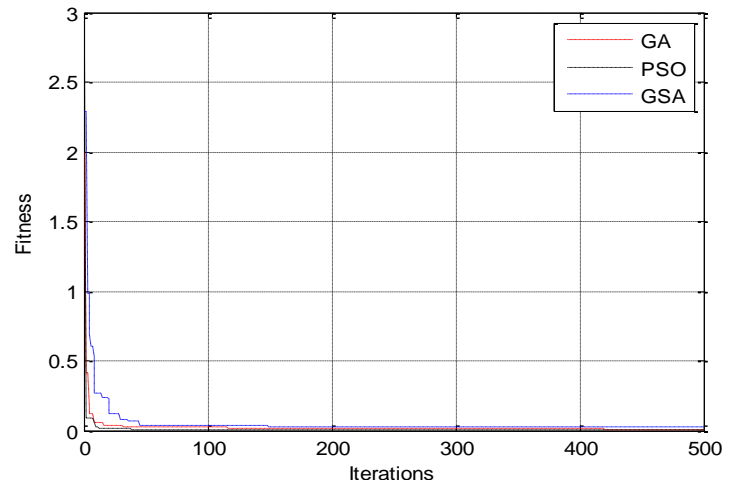

(a)

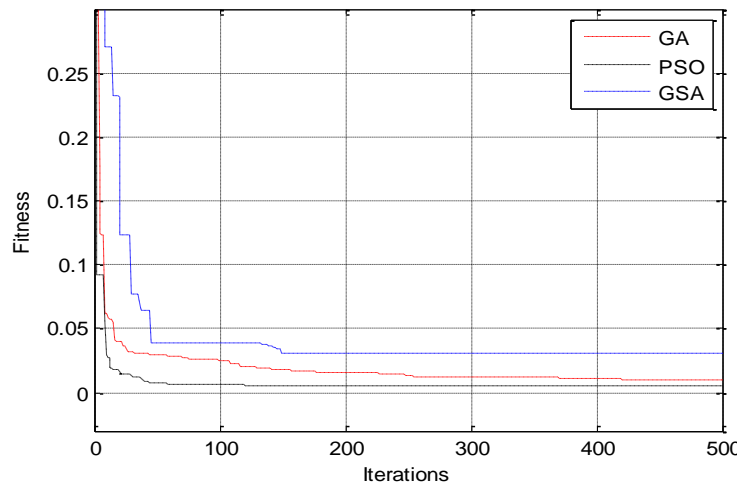

(b)

Figure 11. (a) The performance of the training algorithm for five hidden neuron (b) Zoom at the convergent area of the training algorithm for five hidden neuron

The overall of the modeling performance for the training result is shows in Table 3(a) and Table 3(b). Permeate flux modeling had shown the PSO and GA can be used for the RNN training. Figure 12(a) and Figure 12(b) represents the training result for the permeate flux and TMP using the PSORNN modeling technique.

Generally, the testing results have shown that the result is much improved compared with the three hidden neuron. The PSO and GA training algorithm are able to maintain the accuracy of the model with minimum error increment compared with the training dataset. The testing result reveal similar trend of accuracy with the training result where the PSO algorithm obtains higher accuracy of the model followed with GA and GSA. Table 4(a) and Table 4(b) present the testing performance of the models with the $\% R^{2}$, MSE and MAD performance. The testing result for the permeate flux and TMP were shown in Figure 13(a) and Figure 13(b).

Table 3. (a) Permeate Flux Modeling five hidden Neuron Training Result

\begin{tabular}{cccc}
\hline Method & $\% R^{2}$ & MSE & MAD \\
\hline GA-RNN & 93.00 & 0.0071 & 0.0489 \\
PSO-RNN & 95.26 & 0.0048 & 0.0321 \\
GSA-RNN & 76.10 & 0.0242 & 0.0974 \\
\hline
\end{tabular}

Table 3. (b) TMP Modeling Training five hidden Neuron Result

$\begin{array}{cccc}\text { Method } & \% R^{2} & \text { MSE } & \text { MAD } \\ \text { GA-RNN } & 96.10 & 0.0027 & 0.0349 \\ \text { PSO-RNN } & 98.47 & 0.0011 & 0.0259 \\ \text { GSA-RNN } & 65.12 & 0.0586 & 0.0837\end{array}$



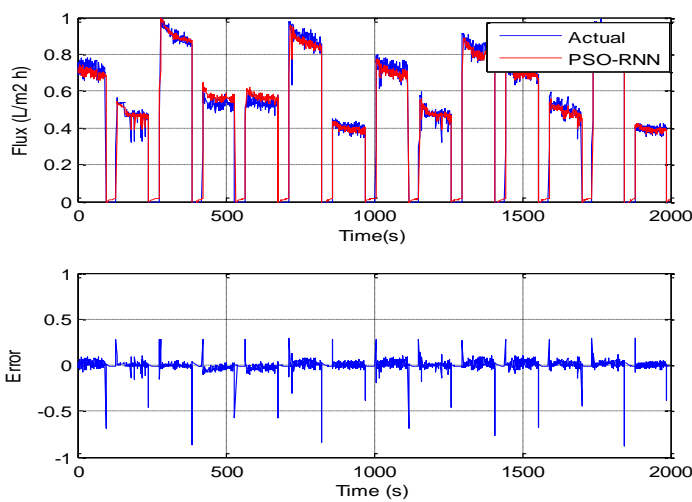

(a)
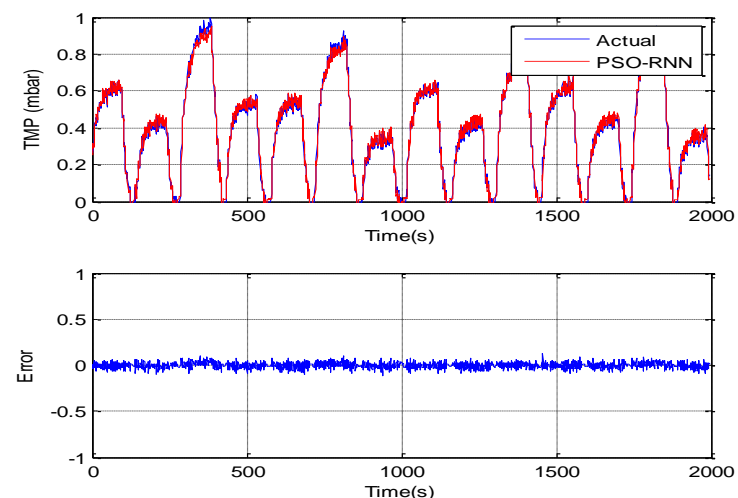

(b)

Figure 12. (a) Permeate Flux modeling training result using RNN-PSO five hidden neuron and the residuals between actual and model (b) TMP modeling training result using RNN-PSO five hidden neuron and the residuals between actual and model

Table 4. (a) Permeate Flux Modeling Testing Result

\begin{tabular}{cccc}
\hline Method & $\% R^{2}$ & MSE & MAD \\
\hline GA-RNN & 93.22 & 0.0066 & 0.0468 \\
PSO-RNN & 94.91 & 0.0049 & 0.0341 \\
GSA-RNN & 74.47 & 0.0248 & 0.0995 \\
\hline
\end{tabular}

Table 4. (b) TMP Modeling Testing Result

\begin{tabular}{cccc}
\hline Method & $\% R^{2}$ & MSE & MAD \\
\hline GA-RNN & 96.68 & 0.0020 & 0.0322 \\
PSO-RNN & 98.46 & $9.4640 \mathrm{e}-004$ & 0.0243 \\
GSA-RNN & 60.43 & 0.0620 & 0.0818 \\
\hline
\end{tabular}
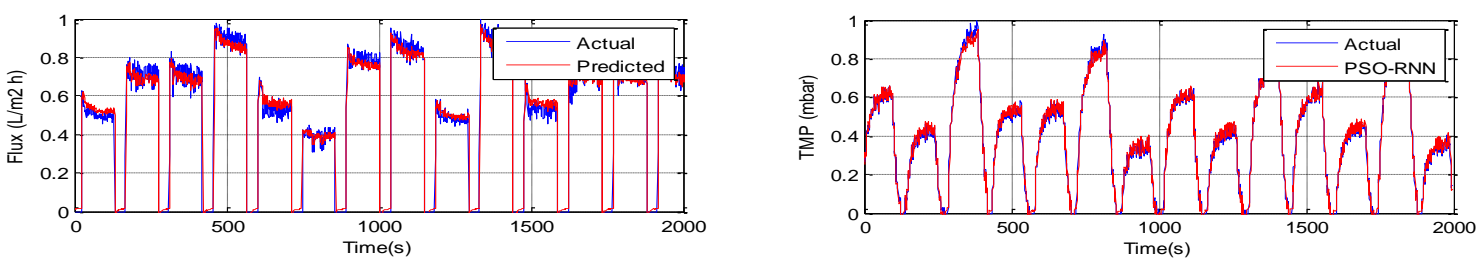

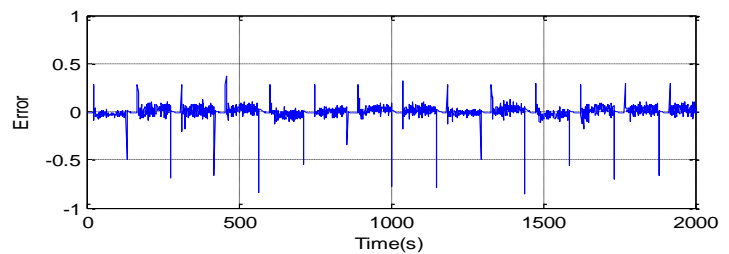

(a)

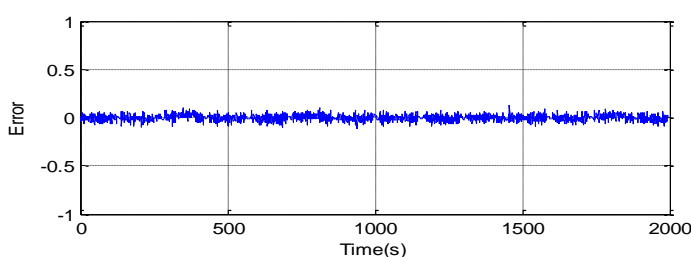

(b)

Figure 13. (a) Permeate Flux modeling testing result using RNN-PSO three hidden neuron and the residuals between actual and model (b): TMP modeling testing result using RNN-PSO three hidden neuron and the residuals between actual and model

\section{CONCLUSION}

This paper presents the dynamic modeling technique for submerged membrane filtration process using RNN with various soft computing techniques. From the result it showed that the training algorithm using soft computing technique is able to produce high accuracy of RNN model. This work compares optimization algorithms of GA, PSO and GSA method to train the RNN model. It is observed that the PSO algorithm is able to gives higher accuracy compared with the others followed with GA and GSA. The 
performance of the PSO and GA algorithm are almost similar especially for the TMP model. Increment from three to five hidden neuron can increase the accuracy of the model, however, it is can also be troublesome for GSA algorithm to find an optimum weights and biases value for the model. Finally this model will be useful in order to do prediction performance and controller design strategy in the future.

\section{ACKNOWLEDGEMENTS}

The authors would like to thank the Research University Grant (GUP) vote 13H70, Universiti Teknologi Malaysia for the financial support. The first author wants to thank the Universiti Teknologi MARA (UiTM) and the MOHE for the TPM-SLAI scholarship

\section{REFERENCES}

[1] S. Judd, The MBR Book Principles and Applications of Membrane Bioreactors in Water and Wastewater Treatment, Second Edi. Elsevier, 2010.

[2] S. Geissler, T. Wintgens, T. Melin, K. Vossenkaul, and C. Kullmann, "Modelling approaches for filtration processes with novel submerged capillary modules in membrane bioreactors for wastewater treatment," Desalination, vol. 178, pp. 125-134, 2005.

[3] A. Aidan, N. Abdel-Jabbar, T. H. Ibrahim, V. Nenov, and F. Mjalli, "Neural network modeling and optimization of scheduling backwash for membrane bioreactor," Clean Technol. Environ. Policy, vol. 10, no. 4, pp. 389-395, Dec. 2007.

[4] T. T. Chow, G. Q. Zhang, Z. Lin, and C. L. Song, "Global optimization of absorption chiller system by genetic algorithm and neural network," Energy Build., vol. 34, pp. 103-109, 2002.

[5] A. R. Pendashteh, a Fakhru'l-Razi, N. Chaibakhsh, L. C. Abdullah, S. S. Madaeni, and Z. Z. Abidin, "Modeling of membrane bioreactor treating hypersaline oily wastewater by artificial neural network.," J. Hazard. Mater., vol. 192, no. 2, pp. 568-75, Aug. 2011.

[6] Wang, Zhen-Hua, Dian-Yao Gong, Xu Li, Guang-Tao Li, and Dian-Hua Zhang, "Prediction of bending force in the hot strip rolling process using artificial neural network and genetic algorithm (ANN-GA)," The International Journal of Advanced Manufacturing Technology, vol. 93, pp. 3325-3338, 2017.

[7] Chatterjee, Sankhadeep, Sarbartha Sarkar, Sirshendu Hore, Nilanjan Dey, Amira S. Ashour, and Valentina E. Balas, "Particle swarm optimization trained neural network for structural failure prediction of multistoried RC buildings," Neural Computing and Applications, vol. 28, no. 8, pp. 2005-2016, 2017.

[8] K. W. Chau, "Application of a PSO-based neural network in analysis of outcomes of construction claims," Autom. Constr., vol. 16, no. 5, pp. 642-646, Aug. 2007.

[9] L. Zhifeng, P. Dan, W. Jianhua, and Y. Shuangxi, "Modelling of Membrane Fouling by PCA-PSOBP Neural Network," 2010 Int. Conf. Comput. Control Ind. Eng., vol. 34, pp. 34-37, 2010.

[10] E. Rashedi, H. Nezamabadi-pour, and S. Saryazdi, "GSA: A Gravitational Search Algorithm," Inf. Sci. (Ny)., vol. 179 , no. 13 , pp. 2232-2248, Jun. 2009.

[11] E. Rashedi, H. Nezamabadi-pour, and S. Saryazdi, "Filter modeling using gravitational search algorithm," Eng. Appl. Artif. Intell., vol. 24, no. 1, pp. 117-122, Feb. 2011.

[12] Li, Chaoshun, Nan Zhang, Xinjie Lai, Jianzhong Zhou, and Yanhe Xu, "Design of a fractional-order PID controller for a pumped storage unit using a gravitational search algorithm based on the Cauchy and Gaussian mutation," Information Sciences, vol. 396, pp. 162-181, 2017.

[13] J. K. Rajesh, S. K. Gupta, G. P. Rangaiah, and A. K. Ray, "Multiobjective Optimization of Steam Reformer Performance Using Genetic Algorithm," Ind. Eng. Chem. Res., vol. 39, no. 3, pp. 706-717, Mar. 2000.

[14] E. Hopper and B. Turton, "A genetic algorithm for a 2D industrial packing problem," Comput. Ind. Eng., vol. 37, no. 1-2, pp. 375-378, Oct. 1999.

\section{BIOGRAPHIES OF AUTHORS}

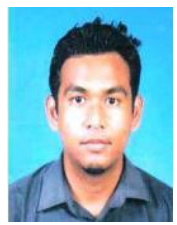

Zakariah Yusuf received his B. Eng. Hons (Electrical) and MSc. Electrical Engineering from Universiti Teknologi MARA, Malaysia in 2007 and 2012 respectively. He is currently working toward his $\mathrm{PhD}$ in process control at the Faculty of Electrical Engineering, Universiti Teknologi Malaysia. He has a few years' experiences in control and instrumentation from various industries. His current research interests include membrane filtration and application of soft computing in the area of process modeling and control

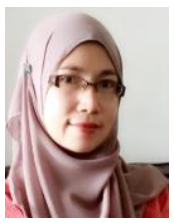

Ir. Dr. Norhaliza Abdul Wahab is currently an Associate Professor at Universiti Teknologi Malaysia (UTM). She is currently the Head Department of Control and Mechatronics Engineering Department at the Faculty of Electrical Engineering, UTM. She completed her PhD in Electrical Engineering majoring in Control in July 2009. She is actively involved in researching and teaching in the field of industrial process control. Her expertise is in modelling and control of industrial process plant. Recently she has 
worked primarily on different types of domestic and industrial wastewater treatment technology towards optimization and energy saving system.

Shafishuhaza Sahlan graduated from University of Sheffield, UK in 2002 with an MEng in Control Systems Engineering. In 2010, she graduated from University of Western Australia upon completion of her studies majoring in Control System Algorithm. Currently, she is working in the Control \& Mechatronics Engineering Department in Faculty of Electrical Engineering in Universiti Teknologi Malaysia, Skudai, Johor as a senior lecturer. Her research interest includes Control Algorithm and its advancement, as well as animal biotechnology. 